\title{
Coordination Polymers, Metal-Organic Frameworks and the Need for Terminology Guidelines
}

Stuart R. Batten ${ }^{a}$, Neil R. Champness ${ }^{b}$, Xiao-Ming Chen ${ }^{c}$, Javier Garcia-Martinez $^{d}$, Susumu Kitagawa ${ }^{e}$ Lars Öhrström ${ }^{f}$, Michael O'Keeffe ${ }^{g}$, Myunghyun Paik Suh ${ }^{h}$ and Jan Reedijk ${ }^{i, j}$

Coordination Polymers (CP) and Metal-Organic Frameworks (MOF) are among the most prolific research areas of inorganic chemistry and crystal engineering in the last 15 years, and yet it still ${ }_{5}$ seems that consensus is lacking about what they really are, or are not.

\section{Background}

Nomenclature and terminology should always create added value. They are tools to incorporate new results into the greater weave of science and enable us to go from the specific to the general. New terminology should also help us to constructively discuss new compounds, ${ }_{10}$ materials and phenomena without having to proceed through endless preambles of definitions.

When two or more sub-disciplines of science find themselves preparing and analysing similar kinds of new materials, an ad hoc terminology often develops from different viewpoints and may not naturally converge into a self-consistent and logical result.

This dual situation occurs for Coordination Polymers (CP) ${ }^{1}$ and Metal-Organic Frameworks ${ }_{15}(\mathrm{MOF})^{2}$, compounds generated in interdisciplinary research fields with their origins in solid state, inorganic and coordination chemistry that have expanded rapidly during the last 15 years. The diversity in both focus and scientific basis of the researchers involved has lead to numerous terminology suggestions and practices for this class of compounds and of several subgroups within them; ${ }^{3}$ additionally, a disquieting number of acronyms are also in use for these materials.

Given the number of publications in the area and the potential applications, this area is now also attracting the interest of the chemical industry ${ }^{4}$, the IUPAC division of Inorganic Chemistry has initiated a project on Coordination polymers and metal organic frameworks: terminology and nomenclature guidelines, ${ }^{5}$ and this communication is a summary of the work of the task group so far and the interactions we have had with scientists in the area.

In terms of strict nomenclature, coordination polymer is approved IUPAC terminology, ${ }^{6}$ but 
only including straight-chain polymers (1D), and not 2D or 3D compounds. Thus in addition to the terminology task, there is also the systematic naming of these compounds that would require attention.

It is evident from the literature that what is logical to one group of scientists would be s unacceptable to another group. At this point one could note that the relation between matter, ("the real world") and the words we use to describe it, is never "perfectly clear". On the contrary, this relation is one of the major unsolved philosophical questions of the $20^{\text {th }}$ century, with the much-debated works of Ludwig Wittgenstein at the centre of the argument. ${ }^{7}$

The purpose of this communication is not to make any official recommendations, (in due ${ }_{10}$ time such recommendations will be published in IUPACs official journal Pure and Applied Chemistry), but rather to spell out the major differences in thinking between various groups, launch a few ideas, and to provide an agenda for further work in the area.

We start to describe the two terms that have been most used, often with overlapping meanings, coordination polymer and metal-organic framework, after a brief introduction to ${ }_{15}$ coordination compounds and polymers.

\section{Coordination compound}

What constitutes a coordination compound? The IUPAC Red Book of inorganic nomenclature from 2005 gives the following definition: "A coordination compound is any ${ }_{20}$ compound that contains a coordination entity. A coordination entity is an ion or neutral molecule that is composed of a central atom, usually that of a metal, to which is attached a surrounding array of atoms or groups of atoms, each which is called a ligand." ${ }^{8}$ We note that this is a very inclusive definition.

\section{Polymer and Coordination polymer}

The term "polymer" was first employed by J.J. Berzelius in 1833 to describe any compound 
that could be formulated as consisting of multiple units of a basic building block. ${ }^{9}$ "Coordination polymer" was first used by Y. Shibata in $1916^{10}$ to describe dimers and trimers of various cobalt(II) ammine nitrates and has been in continuous use in the scientific literature since the 1950 's with what appears to be the first review published in $1964^{11}$. A tutorial review s on "organometallic polymers" from 1981 should also be noted. ${ }^{12}$

The more conventional (organic) polymers were only designated in 1922 when $\mathrm{H}$. Staudinger proposed that the materials previously known as "colloids", such as Bakelite, were in fact monomers held together with covalent bonds, to form what are now known as (organic) polymers ${ }^{9}$.

As even materials commonly known to be polymers as the aforementioned Bakelite, polyethylene, DNA, and cellulose share few, if any, physical properties, the continued use of the IUPAC approved term coordination polymer would seem to be unproblematic as far as properties go. Chapters on "Coordination polymers" can also be found in regular polymer chemistry textbooks, i.e. Carreher's 2010 Introduction to Polymer Chemistry. ${ }^{13}$

However, one may object that "poly-" in English has the meaning of its Greek origin - "more than one"; not "infinity", as we would approach in a good size crystal. An organic polymer with very high degree of polymerization is ultra-high-molecular-weight polyethylene, with up to 200000 repeating units, but usually conventional polymers have much lower degrees of polymerisation. In contrast, a $0.1 \mathrm{~mm}$ cubic crystal of a coordination compound extending infinitely in all three ${ }_{20}$ directions of space by coordination bonds (a $3 \mathrm{D}$ coordination polymer) may easily have $10^{15}$ repeating units (unit cell sides $10 \AA, 1$ molecule per cell), a factor of $10^{9}$ higher. For a corresponding $1 \mathrm{D}$ case, however, the "degree of polymerization" would be less and correspond to the length of a crystal side divided by the unit cell length, in our cases 100000 . This is assuming a perfect, single domain, crystal, something very rare. In practice, therefore, the number of repeating units will be ${ }_{25}$ less.

It is interesting to note that in the early years of crystallography there was a general idea that all crystals were polymers, as many, predominantly English-speaking chemists, denied the existence of non-molecular crystals. For example, the following criticisms appeared in Nature 1927: "Prof. W. L. Bragg asserts that, 'In sodium chloride there appear to be no molecules represented by 
$\mathrm{NaCl}$. '...This statement...is absurd. ... Chemistry is neither chess nor geometry whatever X-ray physics may be."14

The current IUPAC-recommended definitions are as follows:

\section{${ }_{5}$ Polymer $^{15}$}

A substance composed of macromolecules

\section{Polymer molecule (macromolecule) ${ }^{15}$}

A molecule of high relative molecular mass, the structure of which essentially comprises the multiple repetition of units derived, actually or conceptually, from molecules of low relative ${ }_{10}$ molecular mass. ${ }^{\natural}$

\section{Molecular entity ${ }^{16}$}

Any constitutionally or isotopically distinct atom, molecule, ion, ion pair, radical, radical ion, complex, conformer etc., identifiable as a separately distinguishable entity.

One could argue that $\mathrm{AgCl}(\mathrm{s})$ is a coordination polymer because the coordination entities ${ }_{15}\left[\mathrm{AgCl}_{\mathrm{n}}\right]^{(1-\mathrm{n})}$, and possibly also polynuclear species, have individual existence in solution. Even then, this compound hardly meets the criterion of being composed of macromolecules. On the other hand, if we change the chlorides for 4,4'-bipyridine or 1,3-benzenedicarboxylate, can we regard the so formed materials as being composed of macromolecules?

On another conceptual level where we identify polymers with properties like plasticity one ${ }_{20}$ could argue that these are rarely found in crystalline systems. This could be countered with the sub-class of coordination polymers termed "soft". Moreover, if crystallinity is a countercriterion for a polymer, what do we make of the notoriously amorphous vanadiumtetracyanoethylene radical $\left(\left[\mathrm{V}[\mathrm{TCNE}]_{\mathrm{x}} \cdot \mathrm{y}\right.\right.$ solvent) magnetic materials ${ }^{17}$ from the Miller group?

Thus, while in general it is clear that a crystalline material is not necessarily a polymer, the ${ }_{25}$ distinction is perhaps not always easily made.

\section{Metal-organic framework}

The origin of is this term is fairly recent ${ }^{18}$ and has taken on a multitude of meanings with 
numerous scientists proposing more or less converging definitions. In Figure 1 we have depicted the answers to a recent survey undertaken by the present task group where a number of tentative definitions were proposed.

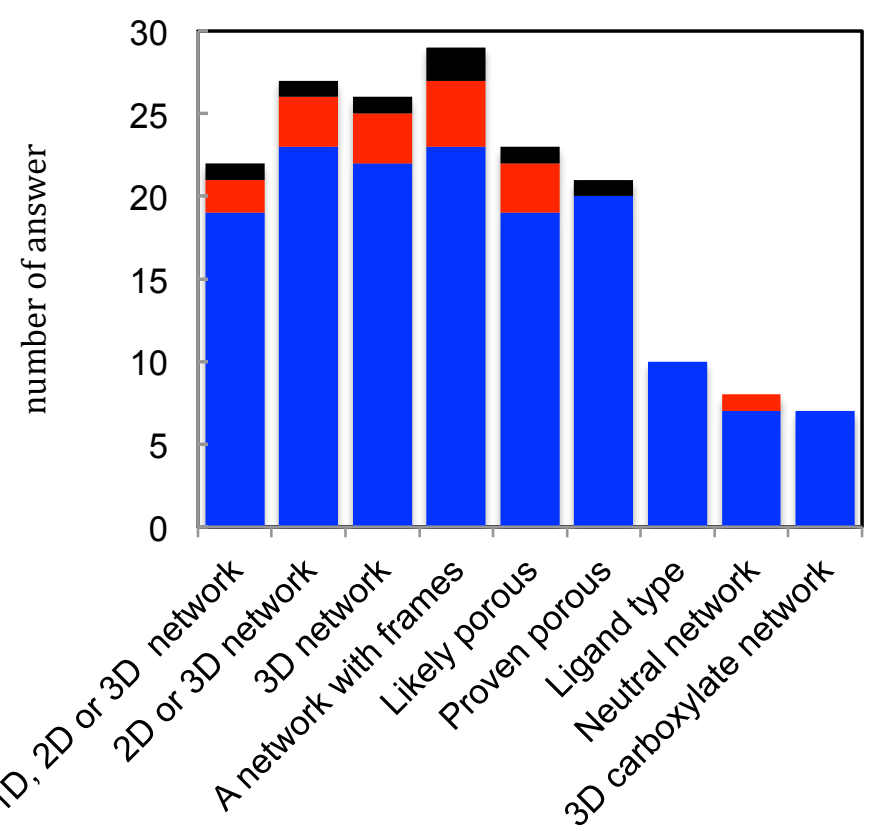

Figure 1. Different definitions of metal-organic frameworks suggested in the questionnaire. Multiple selections were possible and the answers from three subsets have been added: Blue: obtained after postings on the RSC web, the ACS Cryst. Growth \& Design community web and the IUPAC website. Red: from the editorial and advisory boards etc. of Dalton Transactions, CrystEngComm and Crys. Growth \& Design. Black: from contributors to Metal-Organic Frameworks: Design and Application. (MacGillivray, 2010 ${ }^{19}$ )

We do not suggest that nomenclature issues are best resolved by a popular vote; a few things, however, are worth pointing out.

A fairly large minority, 21 out of 91, believe that MOFs need to be proven porous by measuring gas sorption isotherms, but none of the scientists associated with the journals Dalton Transactions, CrystEngComm and Crystal Growth \& Design agree on this. It is also the task ${ }_{15}$ group's standing that such a strict definition would be difficult to enforce and, moreover, would disqualify many materials already labelled as MOFs in the literature from this category.

We also think it is worth noting that only $8 \%$ of the answers indicate that carboxylates is a defining part of a MOF. At the same time, nobody is denying the importance and the critical 
step forward it was for the whole area when these materials started to appear.

\section{"Organic" in metal-organic}

We are not aware of an IUPAC definition of organic, and it is perhaps advantageous that ${ }_{s}$ some terms remain slightly fuzzy. So, we will not attempt to change this situation, thus whether oxalates, trifluoroacetates, cyanides and tetracyanoethylene are considered organic, or inorganic will, be left to the individual chemists' discretion.

\section{Coordination polymer versus MOF}

A number of answers to our survey indicate that $1 \mathrm{D}, 2 \mathrm{D}$ or $3 \mathrm{D}$ coordination polymer covers all possible cases, and that metal-organic framework is a superfluous term and should not be used. However, this is not the opinion of the IUPAC task group. "MOF" is now such a widely employed term it would not just disappear because of an article in Pure and Applied Chemistry. Moreover it has the advantage of it being close to a self-definition. Thus while "coordination ${ }_{15}$ polymer" and "porous coordination polymer" may be easy enough to grasp for chemists, for researchers in closely related disciplines, such as physicists, biologists, and even biochemists, not to speak of the scientific literate among the general public, coordination chemistry is mostly an unknown subject. On the other hand, many in this group can form an intuitive understanding of what you get if you combine metal ions and organic molecules to some kind of framework.

20

A more practical problem is that a large number of groups, predominantly those approaching our subject from the solid-state inorganic side, are not using the term "coordination polymer" and many coordination chemists do not use the term "metal-organic framework", making literature searches more difficult.

We also note that other generic terms are in use such as "hybrid inorganic-organic ${ }_{25}$ materials". We consider these inadequate because they refer only in a very broad, undefined way to some overall composition of the material. 


\section{Coordination network solids}

As the area gains more prominence, and possible industrial applications are in sight, it is also finding its way into general and inorganic chemistry textbooks. For example Chemistry ${ }^{3}$ where, under the heading "Coordination networks" MOF-5 is described as "... an example of a s coordination network, otherwise known as a metal-organic framework. ...many of them are porous...". 20

To avoid confusion and to make it easier for students to relate current research to what they know from undergraduate courses, we need to be proactive. While "coordination" seems entirely reasonable to use at this level, a generic term describing both 2D- and 3D-coordination ${ }_{10}$ polymers namely "coordination network solids" might be useful.

The reasoning is that textbook accounts of the solid state normally start with close packing and metals, move on to ionic solids and then treat network solids such as diamond and quartz. A natural subheading in such a section would then be coordination network solids.

The term coordination network solids can then be seen as a compromise: IUPAC is nomenclature can be adhered to even if coordination polymer is avoided. Metal-organic frameworks will thus be a subclass of coordination network solids, which in its turn is a subclass of coordination polymer, see Figure 2. This rests, however, on a very broad definition of coordination polymer (see discussion above).

An alternative classification that avoids introducing new terms is to adopt a very broad ${ }_{20}$ inclusive definition of metal-organic framework as: "any system that forms a 2D or 3D network with carbon containing ligands bridging mononuclear, polynuclear or 1D coordination entities". See Figure 3.

However, there seems to be some agreement from the surveys that the "frames" in some respect should be "empty", or at least it should be theoretically possible to remove what is in ${ }_{25}$ the cavities.

\section{A hierarchy of solid state coordination chemistry}


Two tentative hierarchies based on the discussion in this communication are presented in Figures 2 and 3. These should, however, not yet be regarded as a final propositions from this IUPAC project.

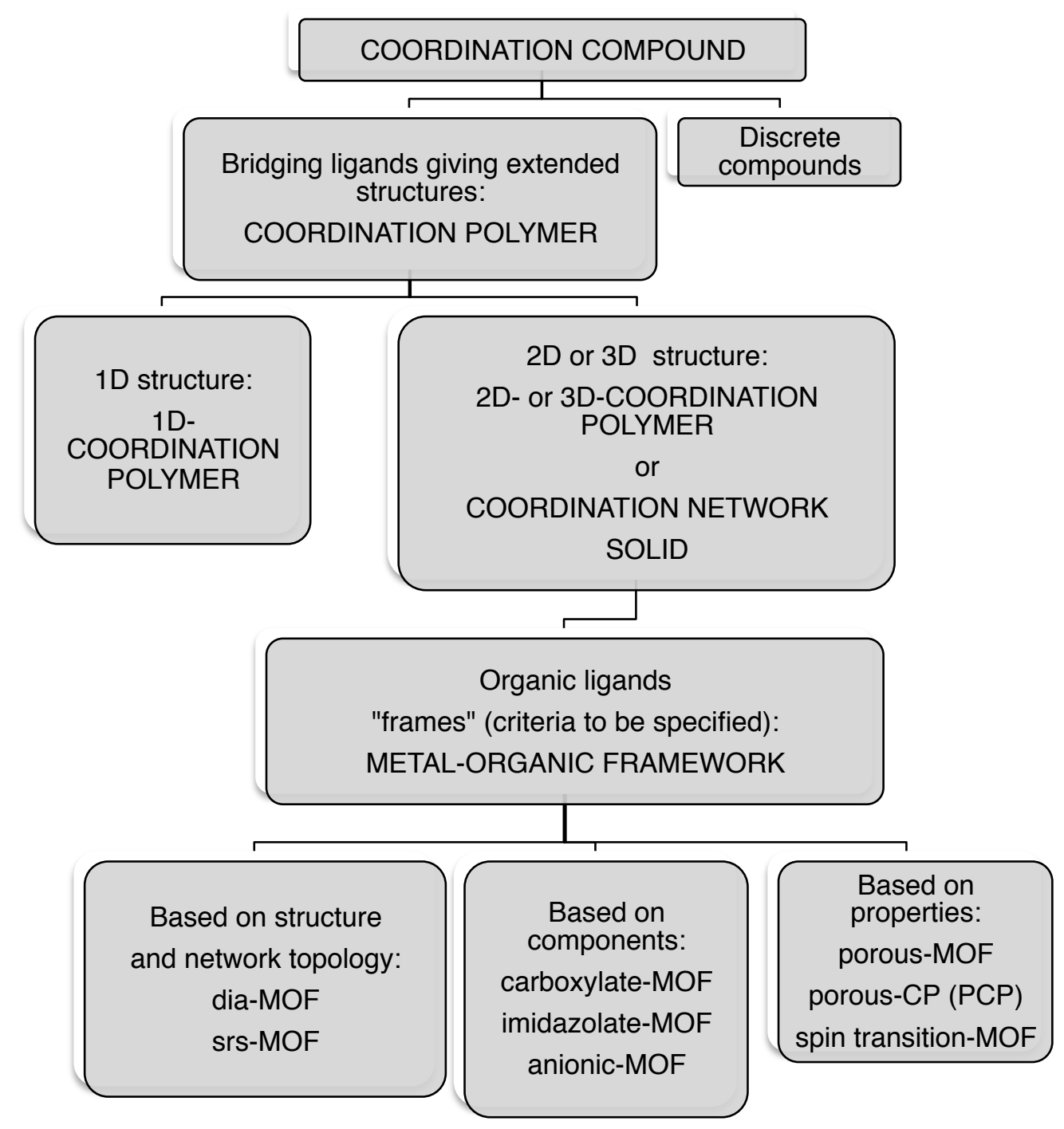

s Figure 2. A tentative hierarchy of coordination polymers and metal-organic frameworks. The bottom descriptors are optional and not mutually exclusive. For an alternative see Figure 3. Three-letter topology codes according to O'Keeffe et al. ${ }^{21}$

For example, there is the question whether a CP needs to have been crystallographically characterised, or if it can even be amorphous? Another point is the term PCP, porous ${ }_{10}$ coordination polymers, in principle these could be based on inorganic ligands and thus not be a subcategory of MOFs. One could also argue for a difference based on if the coordination entities are branching points or merely linkers. 


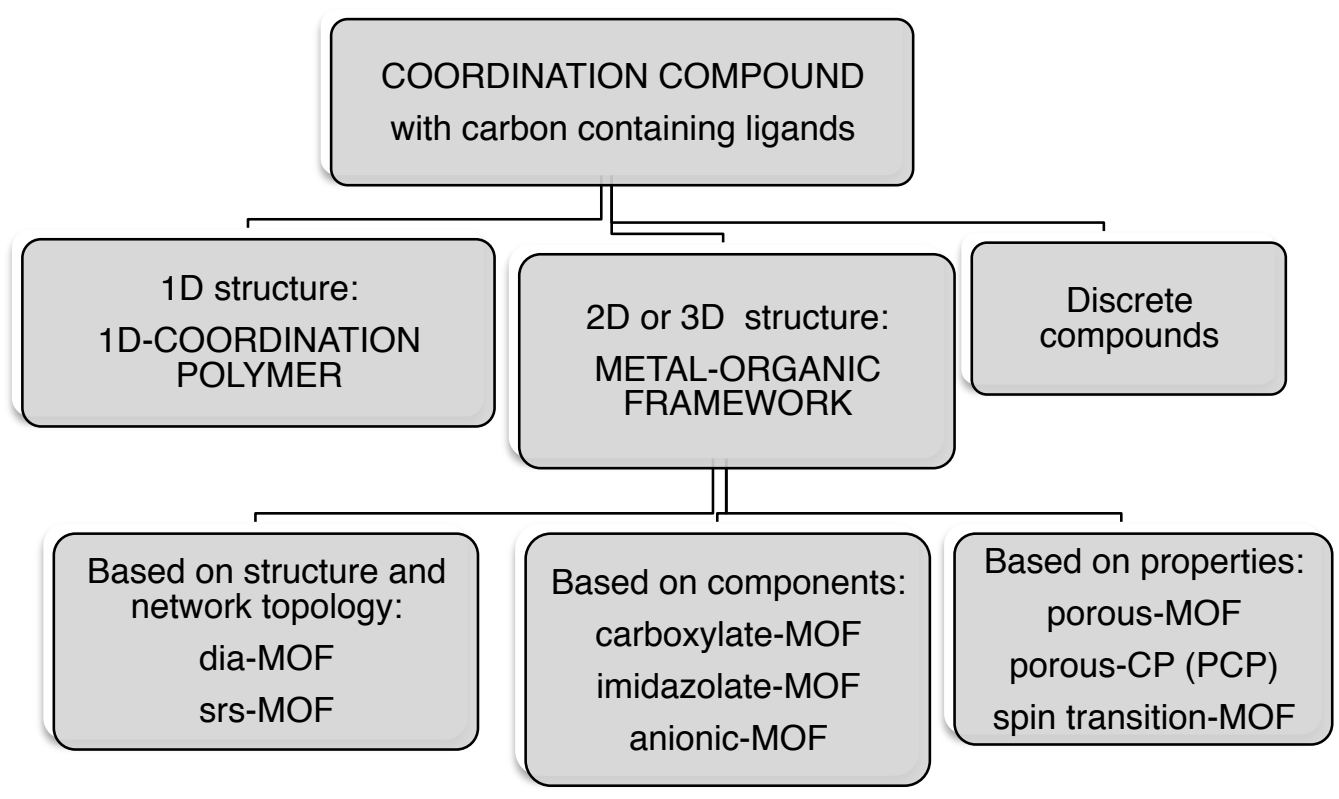

Figure 3. Another tentative hierarchy of coordination polymers and metal-organic frameworks (see also Figure 2). The bottom descriptors are optional and not mutually exclusive. Three-letter topology codes according to O'Keeffe et al. ${ }^{21}$

\section{Subcategories}

The bottom parts of Figure 2 and 3 contain a suggestion for further precision of any MOF. There are a number of other abbreviations in use by various groups, but in our survey we could find no strong support for any other acronym. Instead we propose the use of a number of well${ }_{10}$ defined adjectives describing either the molecular components or the properties of the compound.

\section{Further work}

The task group will likely be inclined to propose the use of network topologies as ${ }_{15}$ descriptors of CP and MOF structures, but as this is a matter closely related to the crystal structure, discussions that also require a dialogue with the International Union of 
Crystallography.

Comments on this text are warmly welcomed (address the task group chairman) and we expect that the discussion will be on-going during 2011-12; the final report is expected to appear in the official IUPAC journal Pure and Applied Chemistry in 2012.

\section{Addresses}

Professor Myunghyun Paik Suh

Department of Chemistry

Seoul National University

Seoul 151-747, South Korea

mpsuh@snu.ac.kr

Dr. Javier Garcia-Martinez

Universidad de Alicante

Departamento de Química Inorgánica

15 Carretera San Vicente del Raspeig s/n

E-03690 Alicante, Spain

j.garcia@ua.es

Professor Susumu Kitagawa

Dept. of Synthetic Chemistry and Biological Chemistry, Kyoto University, Katsura,

Nishikyo-ku, Kyoto, 615-8510, Japan

kitagawa@sbchem.kyoto-u.ac.jp

Dr. Stuart Batten

School of Chemistry, Monash University

Victoria 3800, Australia

25

stuart.batten@sci.monash.edu.au

Prof. Neil Champness

School of Chemistry

University of Nottingham

Nottingham NG7 2RD, UK

30_Neil.Champness@nottingham.ac.uk

Prof. Michael O'Keeffe

Dept. of Chemistry \& Biochemistry,

Arizona State University, Tempe, AZ 85287, USA

mokeeffe@asu.edu

35 Prof. Xiao-Ming Chen

School of Chemistry \& Chemical Engineering

Sun Yat-Sen University

Guangzhou, PR China, 510275

cxm@mail.sysu.edu.cn

40 Prof. Dr. Jan Reedijk

Co-ordination and Bio-Inorganic Chemistry

Leiden Institute of Chemistry 
P.O. Box 9502

2300 RA Leiden

The Netherlands

reedijk@chem.leidenuniv.nl

s Professor Lars Öhrström

Dept. of Chemical and Biological Engineering

Physical Chemistry

Chalmers University of Technology

SE-412 96 Göteborg

10 Sweden

+4631772 2871, ohrstrom@chalmers.se

\section{Notes}

I 1. In many cases, especially for synthetic polymers, a molecule can be regarded as having a high relative molecular mass if the addition or removal of one or a few of the units has a negligible effect on the molecular properties. This statement fails in the case of certain macromolecules for which the properties may be critically dependent on fine details of the molecular structure. ${ }^{15}$

2. If a part or the whole of the molecule has a high relative molecular mass and essentially comprises the multiple repetition of units derived, actually or conceptually, from molecules of low relative molecular mass, it may be described as either macromolecular or polymeric, or by polymer used adjectivally. ${ }^{15}$

\section{${ }_{25}$ References}

1. S. R. Batten, D. R. Turner and M. S. Neville, Coordination Polymers: Design, Analysis and Application, RSC, Cambridge, 2009.

2. (a) J. L. C. Rowsell and O. M. Yaghi, Micropor. Mesopor. Mater., 2004, 73, 3-14, (b) J.

R. Long and O. M. Yaghi, Chem. Soc. Rev., 2009, 38, 1213-1214.

30 3. K. Biradha, A. Ramana and J. J. Vittal, Cryst. Growth Des., 2009, 9, 2969-2970.

4. (a) M. Jacoby, in Chemical \& Engineering News, 2008, vol. 86, pp. 13-16, (b) A. U. Czaja, N. Trukhan and U. Muller, Chem. Soc. Rev., 2009, 38, 1284-1293. 
5. (a) Coordination Polymers and Metal Organic Frameworks: Terminology and Nomenclature Guidelines, http://www.iupac.org/web/ins/2009-012-2-200, (b) L. Öhrström, in Chem. Int., IUPAC, 2010, Jan-Feb, 23.

6. L. G. Donaruma, B. P. Block, K. L. Loening, N. Plate, T. Tsuruta, K. C. Buschbeck, W. H. Powell and J. Reedijk, Pure \& Appl. Chem., 1985, 57, 149-168.

7. L. Wittgenstein, Tractatus Logico-Philosophicu, Harcourt, New York, 1922.

8. N. G. Connelly, T. Damhus, R. M. Hartshorn and A. T. Hutton, eds., Nomenclature of Inorganic Chemistry IUPAC Recommendations 2005, International Union of Pure and Applied Chemistry, The Royal Society of Chemistry, Cambridge, 2005.

109. A. J. Ihde, The Development of Modern Chemistry, Dover Publications, New York, 1984.

10. Y. Shibata, CAN 11:5339, Journal of the College of Science, Imperial University of Tokyo, 1916, 37, 1-17.

11. J. C. J. Bailar, in Preprative Inorganic Reactions, ed. W. L. Jolly, Interscience Publishers, John Wiley \& Sons, New York, 1964, vol. 1, pp. 1-27.

12. C. E. Carraher, J. Chem. Educ., 1981, 58, 921-934.

13. C. E. Carraher Jr., Introduction to Polymer Chemistry 2nd edn., CRC Press, Inc., 2010.

14. H. E. Armstrong, Nature, 1927, 120, 478.

15. A. D. Jenkins, P. Kratochvil, R. F. T. Stepto and U. W. Suter, Pure Appl. Chem., 1996, 68, 2287-2311.

16. M. Nic, J. Jirat and B. Kosata, the IUPAC Compendium of Chemical Terminology (Gold Book), International Union of Pure and Applied Chemistry, 2009.

17. K. I. Pokhodnya, A. J. Epstein and J. S. Miller, Advanced Materials, 2000, 12, 410-+.

18. O. M. Yaghi and H. L. Li, J. Am. Chem. Soc., 1995, 117, 10401-10402.

${ }_{25}$ 19. L. R. MacGillivray, ed., Metal-Organic Frameworks: Design and Application, Wiley, New York, 2010.

20. A. D. Burrows, J. Holman, A. Parsons, G. Pilling and G. Price, Chemistry ${ }^{3}$, Oxford University Press, Oxford, 2009.

21. M. O'Keeffe, M. A. Peskov, S. Ramsden and O. M. Yaghi, Acc. Chem. Res., 2008, 41, 1782-1789. 
TOC entry

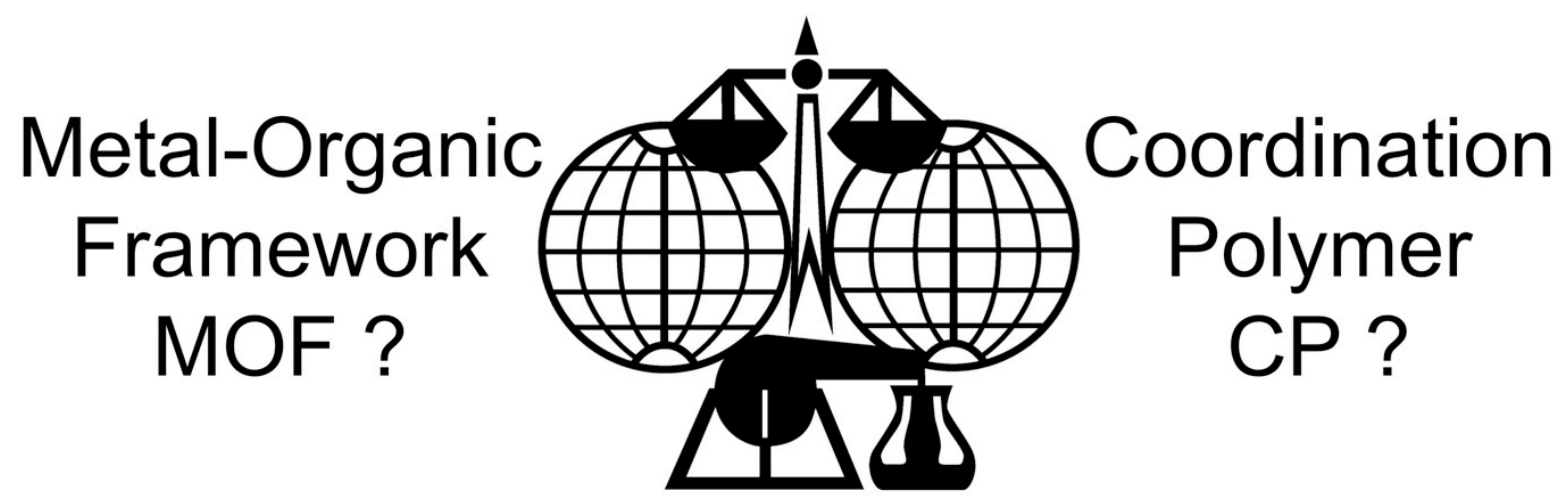

Coordination Polymers (CP) and Metal-Organic Frameworks (MOF) are among the most prolific research areas of crystal engineering in the last 15 years, and the IUPAC Inorganic Chemistry Division has therefore intiated a task group that is currently trying to work out what they really are, or are not. ${ }_{10}$ This is a progress report of the CP/MOF group, more correctly referred to as IUPAC project 2009-0122-200. 